\title{
Improvement of mobile phase in thin-layer chromatography for aflatoxins and analysis of the effect of dichlorvos in aflatoxigenic fungi
}

\section{Keywords}

Aspergillus; chloroform; mobile phase; toluene

Thin-layer chromatography for aflatoxins using toluene as a constituent of a mobile phase worked well and was able to avoid the usage of chloroform. We analyzed the effect of dichlorvos in aflatoxigenic fungi using above mobile phase, and found the difference between two strains of Aspergillus flavus and a strain of $A$. parasiticus.

Aflatoxins (AFs), mainly produced by Aspergillus flavus and A. parasiticus, are carcinogenic mycotoxins with acute hepatotoxicity. AFs exert strong fluorescence under ultraviolet light (365 nm) so that they are detectable by thin-layer chromatography (TLC) simply and easily. Thus far, TLC for AFs adopts chloroform as mobile phases, such as $1.5 \%$ methanol in chloroform ${ }^{1), 2 \text { ), }}$ chloroform:acetone $\left.=90: 10^{3}\right)$, and chloroform: ethyl acetate: $90 \%$ formic acid $=60: 30: 10^{4}$ ).

Chloroform was designated as one of the specified chemical substances in Japan $(2014)^{5)}$ which require the working environment measurement and the reservation of its record for 30 years. Therefore, we examined another solvent applicable to TLC for AFs. After trials, we found that replacement of chloroform with toluene worked well (Supplementary Fig 1. (a); chloroform:ethyl acetate:90\% formic acid $=60: 30: 10$, (b); toluene:ethyl acetate:90\% formic acid $=60: 30: 10$ ). Alternation of $90 \%$ formic acid to acetic acid also worked well as shown in Supplementary Fig 1. (b) and (c). We adopted the last mobile phase (Supplementary Fig 1. (c); toluene: ethyl acetate: acetic acid $=60: 30: 4)$ in further studies. All these mobile-phases were applicable for the separation of two colored intermediates of AFs; versiconal hemiacetal acetate (VHA) and versiconol acetate (VOAc).

Using above mobile phase, we investigated the effect of dichlorvos (DV) on two domestic strains of $A$. flavus. DV is a well-known inhibitor of AF production in A. parasiticus ${ }^{6), 7)}$. In the current study, an A. parasiticus strain NRRL 2999 was used as a positive control for its stable production of both $B$-group $A F s\left(A F B_{1} A F B_{2}\right)$ and G-group $A F s\left(A F G_{1}\right.$ and $A F G_{2}$ ), while an $A$. oryzae strain NBRC 4251 was used as a negative control.

We inoculated an A. parasiticus strain (NRRL 2999), two strains of $A$. flavus (MAFF 111229 and HA9-S1-18)) and an A. oryzae strain (NBRC 4251) on GY2-0.5 agar plate (glucose $2 \%$, yeast extract $0.5 \%$, and agar $2 \%, 15$ $\mathrm{mL} /$ plate) pre-spread with DV in various amounts (80 $\mu \mathrm{g}, 8 \mu \mathrm{g}$, and $0.8 \mu \mathrm{g}$ ).

DV showed the clear inhibition of AFs production in a strain of $A$. parasiticus (NRRL 2999) in dose-dependent manner while a strain of $A$. oryzae (NBRC 4251) did not show any accumulation of AFs, as expected. VHA accumulation in NRRL 2999 was found under higher concentrations of DV (Fig. 1, lanes of $8 \mu \mathrm{g}$ DV and $80 \mu \mathrm{g}$ DV of NRRL 2999).

In contrast, the dose-dependency of DV was not clear in the cases of $A$. flavus strains and the effect of DV was strain dependent. (Fig. 1, lanes of MAFF 111229 and HA9-S1-1).

In summary, DV showed inhibitory effects on AFs production in all strains. There was a difference between species and strains. The TLC method improved here will be applicable for rapid and convenient analysis of AFs.

\section{Masayo Kushiro ${ }^{1}$, Hidemi Hatabayashi ${ }^{1}$, Hiroyuki Nakagawa ${ }^{1,2}$, Kimiko Yabe ${ }^{3}$}

${ }^{1}$ Food Safety Division, Food Research Institute, National Agriculture and Food Research Organization (NARO), 2-1-12 Kannondai, Tsukuba, Ibaraki 305-8642, Japan

${ }^{2}$ Advanced Analysis Center, NARO, 2-1-12 Kannondai, Tsukuba, Ibaraki 305-8642, Japan

${ }^{3}$ Department of Environmental and Food Sciences, Fukui University of Technology, 3-6-1, Gakuen, Fukui-shi, Fukui 910-8505, Japan

\section{Correspondence}

Masayo Kushiro

E-mail: kushirom@affrc.go.jp

(Received January 13, 2017, Revised January 25, 2017, Accepted January 26, 2017)

\section{Acknowledgements}

We thank Dr. Hiromitsu Nakajima for providing us with the standards of VHA and VOAc. We thank Research Project for Improving Food Safety and Animal Health by MAFF, Japan and the Grant from the Tojuro Iijima Foundation for Food Science and Technology, for financial support. 


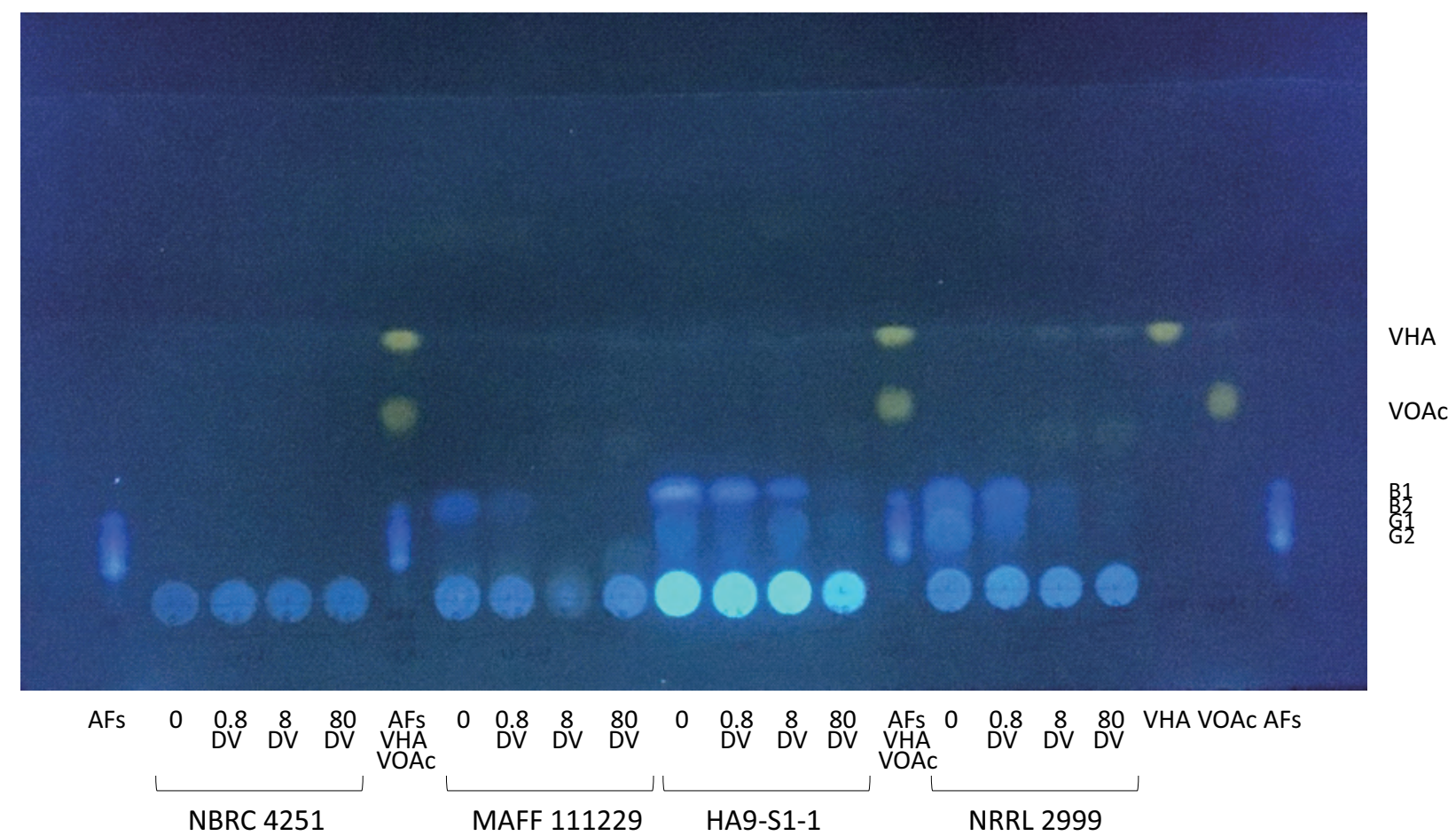

Fig. 1 Thin-layer chromatogram of standard $A F s\left(A F B_{1}, A F B_{2}, A F G_{1}\right.$ and $\left.A F G_{2}\right)$, extract of GY agar (containing 0-80 $\mu \mathrm{g}$ DV each) with A. oryzae NBRC 4251, A. flavus MAFF 111229, A. flavus HA9-S1-1 or A. parasiticus NRRL 2999, VHA and VOAc. On silica gel plate (MERCK Art 5744, SILICA GEL 60 F-254) with mobile phase of toluene:ethyl acetate:acetic acid = 60:30:4.

\section{Supplementary Materials}

Supplementary material may be found in the online version of this article:

Supplementary Fig. 1 Thin-layer chromatogram of standard $A F s\left(\mathrm{AFB}_{1}, \mathrm{AFB}_{2}, \mathrm{AFG}_{1}\right.$ and $\left.A F G_{2}\right)$, and intermediates VHA and VOAc on silica gel plate (MERCK Art 5744, SILICA GEL 60 F-254) with mobile phases of (a) chloroform:ethyl acetate: $90 \%$ formic acid $=60: 30: 10$, (b) toluene:ethyl acetate:90\% formic acid $=60: 30: 10$, (c) toluene:ethyl acetate:acetic acid $=60: 30: 4$.

\section{References}

1) Genest, C., Smith, D. M.: A note on the detection of aflatoxins in peanuts butter. J A O A C, 46, 817-818 (1963)

2) Broadbent, J. H., Cornelius, J. A., Shone, G.: The detection and estimation of aflatoxin in groundnuts and groundnut materials. Part II. Thin-layer chromatographic method. Analyst, 88, 214-216 (1963)

3) Waltking, A. E., Bleffert, G., Kiernan, M.: An improved rapid physicochemical assay method for aflatoxin in peanuts and peanut products. J Am Oil Chem Soc, 45, 880-884 (1968)

4) Yabe, K., Nakamura, H., Ando, Y., Terakado, N., Nakajima, H., Hamasaki, T.: Isolation and characterization of Aspergillus parasiticus mutants with impaired aflatoxin production by impaired aflatoxin production by a novel tip culture method. Appl Environ Microbiol, 54, 2096-2100 (1988)

5) Ministry of Health, Labour and Welfare, Japan [Accessed 13 January 2017]. Amendment of the Ordinance on Prevention of Hazards Due to Specified Chemical Substances, Working Environment Measurement Standards, etc. in November 2014 (Addition of regulations of DDVP and chloroform and nine other substances) (in Japanese) http://www.mhlw.go.jp/stf/seisakunitsuite/bunya/ 0000057700.html

6) Yao, R. C., Hsieh, D. P. H.: Step of dichlorvos inhibition in the pathway of aflatoxin biosynthesis. Appl Microbiol, 28, 52-57 (1974)

7) Yabe, K., Hatabayashi, H., Ikehata, A., Zheng, Y., Kushiro, M.: Development of the dichlorvos-ammonia (DV-AM) method for the visual detection of aflatoxigenic fungi. Appl Microbiol Biotechnol, 99, 10681-10694 (2015)

8) Kushiro, M., Hatabayashi, H., Zheng, Y., Yabe, K.: Application of newly-developed dichlorvoseammonia (DV-AM) method to direct isolation of aflatoxigenic fungi from field soils. Mycoscience, in press 
(a)

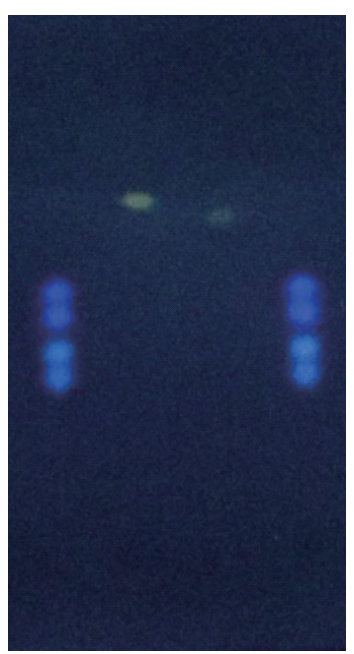

(b)

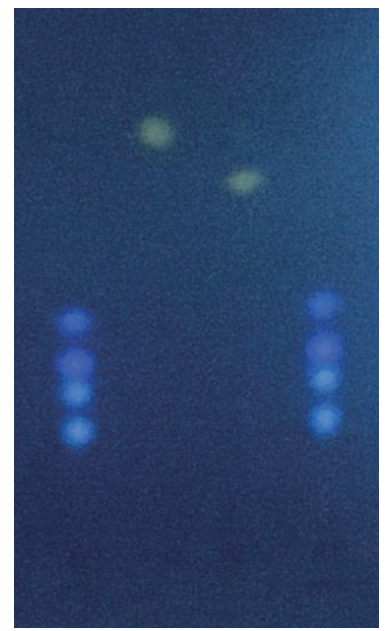

(c)

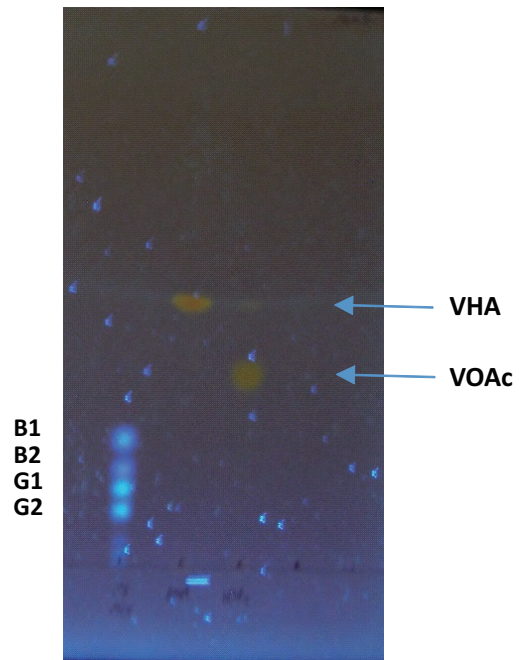

Supplementary Fig 1 Thin-layer chromatogram of standard $A F s\left(A F B_{1}, A F B_{2}, A F G_{1}\right.$ and $A F G_{2}$ ), and intermediates $V H A$ and VOAc on silica gel plate (MERCK Art 5744, SILICA GEL 60 F-254) with mobile phases of (a) chloroform:ethyl acetate:90\% formic acid $=60: 30: 10$, (b) toluene:ethyl acetate:90\% formic acid $=60: 30: 10$, (c) toluene:ethyl acetate:acetic acid $=60: 30: 4$ 